\title{
Descrição do pupário de Raphiocera armata (Wiedemann) (Diptera, Stratiomyidae) da Ilha da Marambaia, Rio de Janeiro, Brasil
}

\author{
José Roberto Pujol-Luz ${ }^{1}$, Roberto de Xerez ${ }^{2}$ \& Gustavo Glória Viana ${ }^{3}$ \\ ${ }^{1}$ Departamento de Zoologia, Instituto de Biologia, Universidade de Brasília. 70910-900 Brasília, Distrito Federal, Brasil. \\ E-mail: jrpujol@unb.br \\ 2 Departamento de Biologia Animal, Instituto de Biologia, Universidade Federal Rural do Rio de Janeiro. \\ 23890-000 Seropédica, Rio de Janeiro,Brasil. E-mail: rdexerez@ufrrj.br \\ ${ }^{3}$ Departamento de Enfermagem, Universidade Estacio de Sá. 28020-740 Campus Campos dos Goitacazes, Rio de Janeiro, \\ Brasil. E-mail gusviana@pop.com.br
}

\begin{abstract}
Description of the puparium of Raphiocera armata (Wiedemann) (Diptera, Stratiomyidae) from Ilha da Marambaia, Rio de Janeiro, Brazil. The puparium of Raphiocera armata (Wiedemann, 1830), based on two larvae reared in the laboratory, collected at Ilha da Marambaia, State of Rio de Janeiro, Brazil is described. This record constitutes the first description of the larvae of a species of the subfamily Raphiocerinae, and associates this group of soldier flies with the aquatic environment, corroborating Woodley's hypothesis of phylogenetic relationships of Raphiocerinae with Stratiomyinae and Nemotelinae, based on the aquatic mode of life of the larvae.

KEY WORDS. Aquatic enviromment, Insecta, larvae, Raphiocerinae, taxonomy.
\end{abstract}

Resumo. O pupário de Raphiocera armata (Wiedemann, 1830) é descrito com base em duas larvas criadas em laboratório, coletadas na Ilha da Marambaia, Estado do Rio de Janeiro, Brasil. Este registro se constitui na primeira descrição da larva de uma espécie da subfamília Raphiocerinae e associa este grupo de moscas com o ambiente aquático, corroborando a hipótese de Woodley de relacionamento filogenético de Raphiocerinae com Stratiomyinae e Nemotelinae baseado no modo de vida aquático das larvas.

PALAVRAS CHAVE. Ambientes aquáticos, Insecta, larvas, Raphiocerinae, taxonomy.

A família Stratiomyidae é composta por 12 subfamílias (Parhadestriinae, Chiromyzinae, Beridinae, Antissinae, Pachygastrinae, Clitellariinae, Sarginae, Chrysochlorininae, Hermetiinae, Raphiocerinae, Stratiomyinae e Nemotelinae). Com exceção de Parhadestriinae e Raphiocerinae, todas as demais subfamílias possuem registros de suas formas imaturas (Woodley 2001). Neste trabalho, descrevemos pela primeira vez o pupário de uma espécie de Raphiocerinae, Raphiocera armata (Wiedemann, 1830), com base em duas larvas coletadas na Ilha da Marambaia e criadas em laboratório.

Wooldey (2001) sugeriu a relação de Raphiocerinae com Stratiomyinae e Nemotelinae em sua hipótese de relacionamento filogenético dos Stratiomyidae com base no número de flagelômeros da antena. Mesmo com total desconhecimento do modo de vida das formas imaturas de Raphiocerinae, o autor incluiu esta subfamília no clado número 6 de sua filogenia, que é sustentado pelo estado apomórfico "larvas aquáticas", onde assinala que todas as larvas conhecidas de Stratiomyinae e Nemotelinae são aquáticas ou associadas com habitats aquáticos (Roskosny 1982, Woodley 2001).

Larvas de Stratiomyidae podem ser agrupadas em dois grupos ecológicos distintos: terrestres e aquáticos (RoskosNy 1997). As larvas terrestres estão associadas principalmente à decomposição de matéria orgânica vegetal e animal em sistemas florestais (e.g. Beridinae, Sarginae, Hermetiinae, Clitellariinae), mas destacam-se também os grupos especializados em viver sob a casca de árvores mortas ou apodrecidas no solo (Pachygastrinae) ou em raízes de plantas vivas (Chiromyzinae). As larvas aquáticas das subfamílias Nemotelinae e Stratiomyinae são encontradas praticamente em todos os tipos de coleções de águas continentais e estão associadas principalmente à vegetação aquática ou ao substrato não consolidado do fundo de rios e lagoas. Algumas espécies podem ser encontradas em regiões pantanosas ou em coleções de água com altos graus de salinidade; outras possuem especializações morfológicas para o modo de vida higropétrico em ambientes lóticos (Roskosny 1997).

No Brasil, os únicos registros de larvas aquáticas são encontrados em um manuscrito não publicado de Newton Dias dos Santos de 1950, onde são descritos hábitos de vida higropétricos de três espécies da subfamília Stratiomyinae no Estado do Rio de Janeiro.

Revista Brasileira de Zoologia 21 (4): 995-999, dezembro 2004 
Raphiocerinae compreende um grupo de moscas essencialmente Neotropical, exceto por Rhaphioceroides pendleburyi Brunetti, 1927 da Tailândia e Timorimyia bidentata Frey, 1934 do Timor. Os adultos são facilmente reconhecidos pela forma alongada do corpo e pelos padrões cromáticos compostos por faixas e manchas circulares, bem definidos e característicos principalmente na cabeça e no tórax, além de possuírem o palpo uni-segmentado e ausência de pilosidade na pós-tégula. Atualmente são reconhecidas 31 espécies na região Neotropical, distribuídas em 21 gêneros (Tab. I) (Pimentel \& Pujol-Luz 2000, 2001, 2002a, b, WoOdLey 2001).

Raphiocera Macquart, 1834 é composto por quatro espécies (Pimentel \& Pujol-Luz 2002b): Raphiocera armata, R. hoplistes (Wiedemann, 1830), R. hortulana (Wiedemann, 1830) e $R$. papaveroi Pimentel \& Pujol-Luz, 2002. A larva de $R$. armata foi coletada na Ilha da Marambaia no litoral sul do Estado do Rio de Janeiro, sobre um tronco em decomposição dentro de uma pequena floresta remanescente de mata atlântica, na área construída do Centro de Adestramento da Ilha da Marambaia do Corpo de Fuzileiros Navais da Marinha do Brasil, a cerca de $3 \mathrm{~m}$ de um riacho que sofre forte influência da água salgada das marés altas na ilha.

Apesar de a larva de $R$. armata ter sido coletada sobre um tronco de árvore em decomposição em um ambiente terrestre, a presença característica em larvas aquáticas (Roskosny 1982) de uma fileira de cerdas hidrófugas plumosas relativamente longas na margem do espiráculo posterior, que nesta espécie é ventral, corrobora a hipótese de Woodley (2001) de agrupar os Raphiocerinae com Stratiomyinae e Nemotelinae com base no habitat aquático das larvas. As larvas de R. armata foram criadas em laboratório até a emergência do adulto, em uma placa de Petri seguindo a mesma metodologia utilizada para a criação de larvas terrestres de Stratiomyidae (Pujol-Luz \& Xerez 1999, PujolLuZ \& Leite 2001, Viana et al. 2003). O material estudado está depositado na Coleção Entomológica do Departamento de Zoologia do Instituto de Biologia da Universidade de Brasília.

São consideradas características distintivas de $R$. armata, em relação às espécies de Stratiomyinae e Nemotelinae conhecidas, a associação dos seguintes caracteres: espiráculo posterior do oitavo segmento do abdome com abertura ventral, presença de tufos de cerdas ventrais digitiformes (tetrâmeras) e plumosas na cabeça e cerdas ventrais do abdome lamelares e franjadas.

\section{Raphiocera armata}

Pupário. Comprimento total $14 \mathrm{~mm}$ (Figs 1 e 2). Corpo cilíndrico, com três segmentos torácicos e oito segmentos abdominais. Duas faixas longitudinais em "zig zag" de cor mais escura que se encontram na região mediana em vista dorsal e são paralelas, em vista ventral, que se iniciam no primeiro segmento torácico e terminam no último segmento abdominal. Segmentos do corpo de forma e tamanho variáveis. Último segmento abdominal de forma quadrangular com um tufo de cerdas partindo do espiráculo respiratório posterior. Cerdas dos
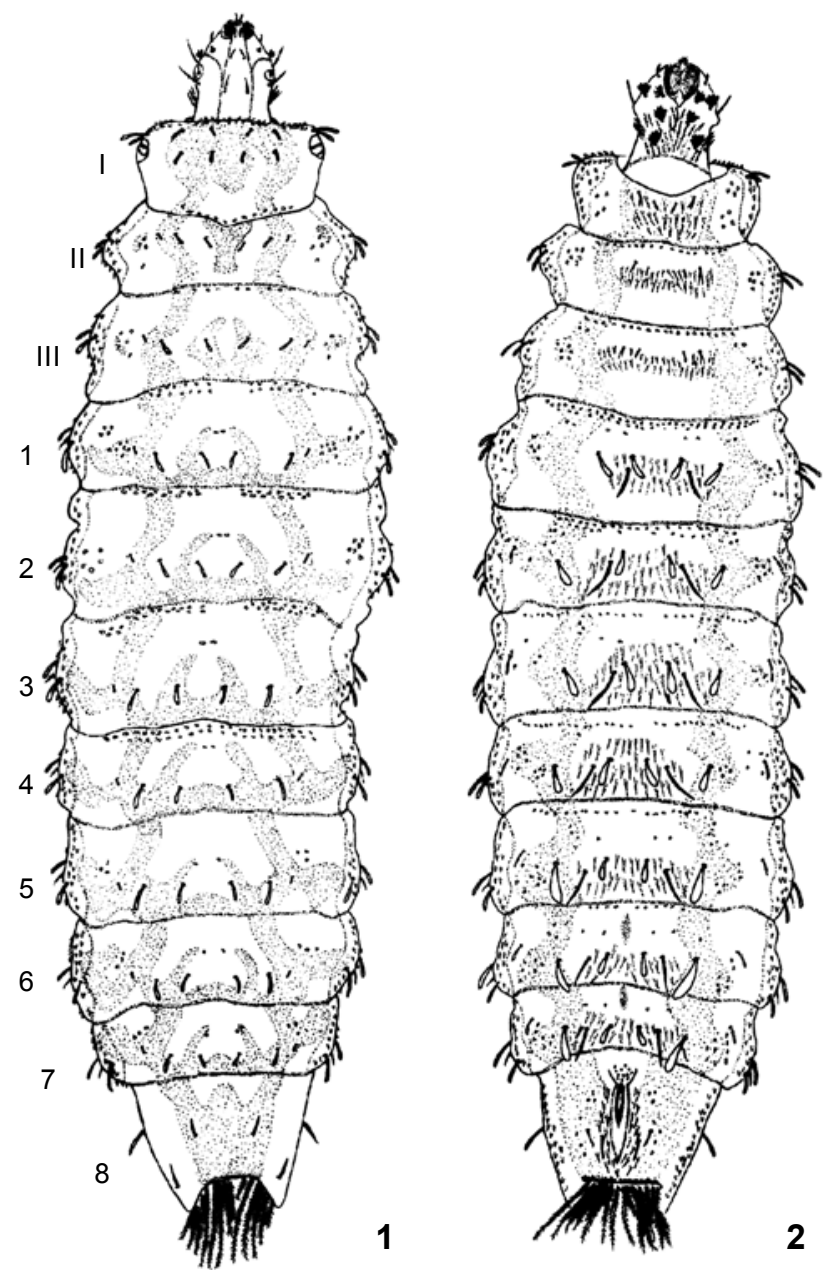

Figuras 1-2. Habitus de Raphiocera armata (Wiedemann) (comprimento total $14 \mathrm{~mm}$ ). 1-vista dorsal, 2- vista ventral. Legendas: I-III (tórax), 1-8 (abdômen).

segmentos abdominais em vista ventral variando no formato, existindo cerdas lamelares franjadas, clavadas e filiformes. Corpo em algumas regiões recoberto por ornamentações cuticulares de carbonato de cálcio típicas das larvas de Stratiomyidae.

Cabeça. Curta, projetada anteriormente, antena curta localizada dorsalmente, entre as cerdas Lb2 e Cf1; olhos pouco proeminentes na região mediana da cápsula cefálica. Quetotaxia em vista dorsal (Fig. 3): dois pares de cerdas labrais (Lb), o primeiro (Lb1) setiforme e o segundo (Lb2) formando um tufo plumoso; dois pares de cerdas clípeofrontais (Cf), o primeiro (Cf1) bifurcado e curto e o segundo (Cf2) setiforme e longo, um par de cerdas dorsolaterais (Dl) situadas acima dos olhos e um par de cerdas laterais (L) localizada abaixo dos olhos. Quetotaxia em vista ventral (Fig. 4): três pares de cerdas ventrais (V) diferenciadas, sendo que V1 e V2 formam tufos plumosos e V3

Revista Brasileira de Zoologia 21 (4): 995-999, dezembro 2004 

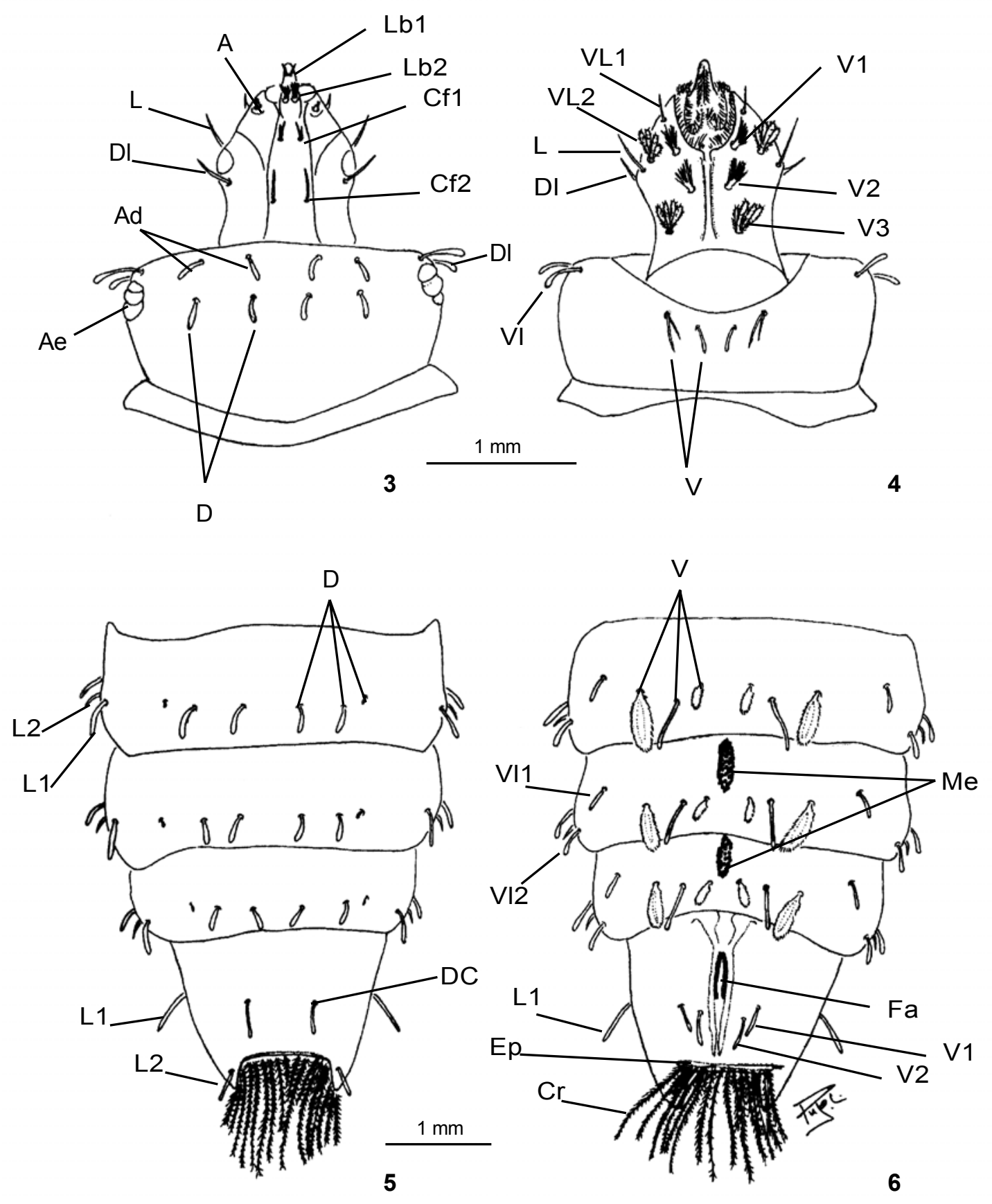

Figuras 3-6. Raphiocera armata, pupa. (3-4) Cabeça e protórax: (3) vista dorsal, (4) vista ventral; (5-6) abdômen: (5) vista dorsal, (6) vista ventral. (A) Antena, (Ad) cerdas anterodorsais, (Ae) espiráculo anterior, (Cf) cerdas clipeofrontais, (Cr) cerdas respiratórias, (D) cerdas dorsais, (DC) cerdas dorsocentrais, (DI) cerdas dorsolaterais, (Ep) espiráculo posterior, (Fa) fenda anal, (L) cerdas laterais, (Me) macha esternal, $(\mathrm{V})$ cerdas ventrais, $(\mathrm{Vl})$ cerdas ventrolaterais.

digitiformes e tetrâmeras, dois pares de cerdas ventrolaterais (Vl) sendo Vl1 setiforme e Vl2 digitiforme e tetrâmeras.

Tórax. Primeiro segmento torácico mais estreito do que os outros (Figs 1 e 2). Em vista dorsal (Fig. 4) com um par de cerdas dorsolaterais (Dl), dois pares de cerdas anterodorsais (Ad), dois pares de cerdas dorsais (D), espiráculo anterior (Ea) pre- 
Tabela I. Número de gêneros e espécies de Raphiocerinae na Região Neotropical e no Brasil (modificado de WoodLEY 2001).

\begin{tabular}{|c|c|c|}
\hline Tribos & Gêneros neotropicais & Número de espécies \\
\hline \multirow[t]{4}{*}{ Analcocerini } & Analcoceroides Hollis, 1963 & 1 (México) \\
\hline & Analcocerus Loew, 1855 & 3 (Brasil) \\
\hline & Anisoscapus McFadden, 1970 & 2 (México) \\
\hline & Neanalcocerus James, 1943 & 1 (México) \\
\hline \multirow[t]{17}{*}{ Raphiocerini } & Brachythrix McFadden, 1970 & 1 (México) \\
\hline & Cyclophleps James, 1943 & 1 (Peru) \\
\hline & Dactylothrix Pimentel \& Pujol-Luz, 2001 & 1 (Equador) \\
\hline & Dicamptocrana Frey, 1943 & 1 (Paraguai) \\
\hline & Dicranophora Macquart, 1834 & 4 (Guiana, Guiana Francesa, Brasil, Paraguai) \\
\hline & Dolichothrix McFadden, 1970 & 1 (México) \\
\hline & Heptozus Lindner, 1949 & 2 (Panamá, Equador) \\
\hline & Histiodroma Schiner, 1868 & 1 (Brasil) \\
\hline & Hoplistes Macquart, 1834 & 1 (Brasil) \\
\hline & Lysozus Enderlein, 1914 & 1 (Colômbia) \\
\hline & Neoraphiocera Pimentel \& Pujol-Luz, 2001 & 1 (Brasil) \\
\hline & Pezodontina Lindner, 1949 & 1 (Brasil, Guiana) \\
\hline & Phanerozus James, 1966 & 1 (Panamá, Costa Rica) \\
\hline & Pseudocyclophleps Pimentel \& Pujol-Luz, 2001 & 1 (Peru) \\
\hline & Pseudohistiodroma Pimentel \& Pujol-Luz, 2001 & 1 (Brasil) \\
\hline & Raphiocera Macquart, 1834 & 4 (Brasil) \\
\hline & Rondonocera Pimentel \& Pujol-Luz, 2001 & 1 (Brasil) \\
\hline
\end{tabular}

sente no protórax. Segundo e terceiro segmentos torácicos (Fig. 1) com três pares de cerdas dorsais (D) um par de cerdas dorsolaterais (Dl). Em vista ventral (Fig. 2), os três segmentos torácicos apresentam dois pares de cerdas ventrais $(\mathrm{V})$, sendo que o par mais externo é bifrucado e um par de cerdas ventrolaterais (Vl) (Fig. 4). Toda a região mediana dos segmentos torácicos em vista ventral é recoberta por um conjunto de pequenas cerdas piliformes (Fig. 2).

Abdômen. Do primeiro ao sétimo segmentos abdominais apresentam em vista dorsal a seguinte quetotaxia (Figs 1, 2, 5 e 6): três pares de cerdas dorsais (D) sendo o par mais externo mais curto do que os dois pares mais internos. Em vista lateral apresenta dois pares de cerdas laterais (L). Oitavo segmento longo (Figs 5 e 6) e mais estreito que os anteriores, formando dois processos distais, com um par de cerdas dorsocentrais (Dc), e dois pares de cerdas laterais (L). Em vista ventral (Fig. 6) com três pares de cerdas ventrais $(\mathrm{V})$, o mais interno (V1) menor que os adjacentes, o par mais interno (V1) e o mais externo (V3) tem forma lamelar e são plumosos, o par mediano (V2) tem a forma afilada; com dois pares de cerdas ventrolaterais (Vl). Mancha esternal presente no sexto e sétimo segmentos (Fig. 6), composta por um mosaico de inúmeras células esféricas diferenciadas daquelas impregnadas por carbonato de cálcio. Oitavo segmento (Fig. 6) com dois pares de cerdas ventrais (V) e um par de cerdas laterais (L). Fenda anal (Fa) abrindo-se longitudinalmente, com bordos internos margeados por densa pilosidade. Espiráculo posterior abrindo-se na região ventral, por onde saem um conjunto de cerdas respiratórias (Fig. 6).

Material examinado. BRASIL, Rio de Janeiro: Mangaratiba (Ilha

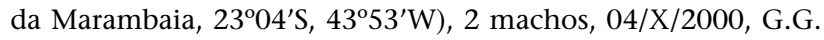
Viana leg. (emergências dos adultos: 05/X/2000 e 19/X/2000).

\section{AGRADECIMENTOS}

Ao Comandante do Centro de Adestramento da Ilha da Marambaia do Corpo de Fuzileiros Navais da Marinha do Brasil, pelas facilidades oferecidas para o desenvolvimento deste trabalho. Ao Conselho Nacional de Desenvolvimento Científico e Tecnológico pelo apoio ao projeto de pesquisa (CNPq/ 300265/96-4).

\section{REFERÊNCIAS BIBLIOGRÁFICAS}

Pimentel, T. \& J.R. Pujol-Luz. 2000. Os gêneros de Raphiocerinae (Diptera, Stratiomyidae) do Brasil e algumas espécies da América do Sul. Parte 1 - A Tribo Analcocerini (sensu Enderlein, 1914). Contribuições avulsas sobre a História Natural do Brasil, Série Zoologia, Seropédica, 23: 1-18.

. 2001. Os gêneros de Raphiocerinae (Diptera, Stratiomyidae) do Brasil e algumas espécies da América do Sul. Parte 2 - A Tribo Raphiocerini (sensu Schiner). Contri-

Revista Brasileira de Zoologia 21 (4): 995-999, dezembro 2004 
buições avulsas sobre a História Natural do Brasil, Série Zoologia, Seropédica, 33: 1-31.

. 2002a. Insecta-Diptera-Stratiomyidae (Subfamília Raphiocerinae). Fauna da Amazônia Brasileira, Belém, 12: 1-3.

2002b. A new species of the genus Raphiocera (Diptera: Stratiomyidae) from Santa Catarina, Brazil. Studia Dipterologica, Halle, 9: 513-517. [2003].

Pujol-Luz, J.R. \& F.M. Leite. 2001. Descrição do ultimo ínstar larval e do pupário de Ptecticus testaceus (Fabr.) (Diptera: Stratiomyidae). Neotropical Entomology, Londrina, 30 (4): 587-591.

Pujol-Luz, J.R. \& R. DE Xerez. 1999. The larva of Chalcidomorphina aurata Enderlein 1914 (Diptera: Stratiomyidae) from "Ilha da Marambaia", Rio de Janeiro, Brasil. Proceedings of the
Entomological Society of Washington 101 (2): 295-299.

Roskosny, R. 1982. A biosystematics study of the European Stratiomyidae (Diptera). Volume 1. Introduction, Beridinae, Sarginae and Stratiomyinae. London, Dr. W. Junk, VIII+401.

1997. Diptera Stratiomyidae, Soldier Flies, p. 321332. In: A.N. NiLsson (Ed.). Aquatic Insects of North Europe - a taxonomic handbook. Stenstrup, Apollo Books, vol. 2, 440p.

Viana, G.G.; J.R. Pujol-Luz \& R de Xerez. 2003. Descrição da larva e do pupário de Auloceromyia vespiformis Lindner (Diptera: Stratiomyidae, Clitellariinae). Neotropical Entomology, Londrina, 32 (1): 69-74.

Woodley, N.E. 2001. A World Catalog of the Stratiomyidae (Insecta: Diptera). Myia, Washington, 11: 1-473.

Recebido em 13.IV.2004; aceito em 19.XI.2004. 